\title{
Renting of Car based on IoT
}

\author{
Srivatsa Ransubhe ${ }^{1}$, Aishwarya Gire ${ }^{2}$, Shweta Bhadale ${ }^{3}$, Gauri Shewale ${ }^{4}$, Prof. Amrin Sheikh ${ }^{5}$ \\ Computer Department, Keystone School of Engineering, Pune, India $a^{1,2,3,4,5}$
}

\begin{abstract}
In this paper the Application giving out outputs to the user for Nearest Available Car like AC and NON AC. The System is highly reliable as it uses Google map API which are very accurate for User Tracking and same goes for the User to travel his Ride.It also allows cities to develop fully integrated multimodal smart transportation systems. Owner will Display his car whenever he didn't required to use as well as Track his car using GPS Tracking System. The User has options to select for the places he wants to visit for instance with all Details of Car; the system will ask whether he is searching for the current locality or some other place. The System is very flexible in changing places and makes use of Google maps to display places if the user wishes to within particular distance mentioned by the user considering a fence of geo locations.
\end{abstract}

Keywords: Smart car, GPS, GSM Module

\section{INTRODUCTION}

Car booking service is a important transport service provided by the various transport operators in a particular city. Most of the people use car service for their daily need. More and more taxi companies are looking for integrated taxi booking systems as it makes life much easier for the customer as well for the company. Most of the existing online cabs booking systems use the centralized approach to search, find, and book the car. In this paper we are implementing the smart car for people. For travelling with Air conditioner or non -air conditioner. Using the GPS and GSM module. Using application admin can register and registration is successful then login the application. Similarly the user can register the application and registration is successful then login the application. user can select the area and travel in smart car. And as per the user choice they on the Air conditioner or not. Then generate the bill . If user go to the out of area then admin off the car .as well as user can see the Car speed.

\section{LITERATURE SURVEY}

[1]"Vehicle Delay Series Forecast Based on Trajectories Of GPS Tracked Cabs."Wenjuan Cui', Danhuai Guo1*I Scientific Data Center, Computer Network Information Center, Chinese Academy of Sciences, Beijing, China .

This paper has defined vehicle delay series which can evaluate LOS of intersection based on drivers' direct experience. We take advantage of large scale of but coarse-grained trajectories data of tracked cabs for rapid vehicle delay series calculation. The kNN anomaly detection algorithm is used on raw vehicle delay series. In pursuit of acquiring criteria for evaluating the performance, we compare several dominating and sophisticated time series analysis algorithms including ARIMA, DSHW, BATS and TBATS. Facing the challenges of outliers aroused from vehicle scarcity, we propose a new Robust TBATS algorithm. Experiments show that our proposed Robust TBA TS outperforms others significantly and is suitable for automatically online forecast of vehicle delay series. Moreover, we suppose several applications of vehicle delay series forecast and demonstrate one of them in route planning.

[2]“Development of Multiple Tracking System for Smart VIP Car Placement and Monitoring."Nizomjon Khajievt, Chol-U Lee2, Kyung-Sook Kim3, Seung-Ho Kim4, Ryum-Duck Oh5 1,2,5 Dept of Information convergence, Korea National University of Transportation 50, Daehak-ro, Daesowon-myeon, Chungju-si, Chungcheongbuk-do 27469.

The system benefits from smart parking go well beyond avoiding the unnecessary circling of city blocks. It also allows cities to develop fully integrated multimodal smart transportation systems Conclusively, this paper is extremely significant for new researchers in the innovation of new techniques to manage the problems which are faced by drivers nowadays. Real-time parking lots streaming through Android application are highly recommended in which will easily help the drivers to allocate the vacant parking lots. It will make the management of the parking spaces effectively, by eliminating the need of manual labour work. 
Vol. 8, Issue 1, January 2019

[3]“Intelligent Ridesharing System for Taxi to Reduce Cab Fee." Jian-Pan Li, Gwo-Jiun Homg, Sheng-Tzong Cheng, Chen-Fei Chen.

In this paper, we have proposed a taxi-sharing recommendation mechanism for both taxis and passengers, which combines a non-cooperative game model to solve the competition among taxis in need of route recommendations. Based on the historical intonation of taxis and passengers, we built time-dependent R-Trees to discover popular locations so that a server can suggest routes and locations using these R-Trees.

[4]"Design and Implementation of an Intelligent (Cab Service System)."Amar Nath, Ankit Khandelwal, Akul Kanojia, Ishitva Minocha, Rajdeep Niyogi

In this paper, we designed an intelligent agent based distributed approach for taxicab booking system. The proposed approach is able to deter the single point of failure, and utilize the local information of the different region of the city to improve the cab availability. Furthermore, to incorporate the bargaining facility and cabs driver participation, we designed the distributed algorithms (see Algorithm 1 and 2). One of the proposed distributed algorithms (Algorithm 2) is implemented by using JADE framework. As part of our ongoing and future work, we would implement our proposed system for real cab booking system.

[5]S"Robust forecasting with exponential and Holt-Winters smoothing, ". Gelper, R. Fried, and C. Croux, Journal of Forecasting, vol. 29, pp. 285- 300, 2010.

Robust versions of the exponential and Holt-Winters smoothing method for forecasting are presented. They are suitable for forecasting univariate time series in the presence of outliers. The robust exponential and Holt-Winters smoothing methods are presented as recursive updating schemes that apply the standard technique to pre-cleaned data. Both the update equation and the selection of the smoothing parameters are robustified. A simulation study compares the robust and classical forecasts. The presented method is found to have good forecast performance for time series with and without outliers, as well as for fat tailed time series and under model misspecification. The method is illustrated using real data incorporating a trend and seasonal effects.

\section{PROPOSE SYSTEM}

In Proposed system we are analysing Smart car for people. In that Admin can register the Android application and registration is successful then login the application. Similarly user can register the same application and registration is successful then login the application. User using the android application can search the near smart car. Then using GPS they pick to user and User select the area and Bill is generate and pay the bill using android application and travelling with smart car. If user choice they can on the Air conditioner or not. Then user send the request to admin. Then admin accept the request and as per air conditioner charge they generate the bill and user can on the Air conditioner and reach to selected area .If user go to another area then admin off the smart car.

\section{SYSTEM ARCHITECTURE}

Following diagram is our system's architecture diagram:

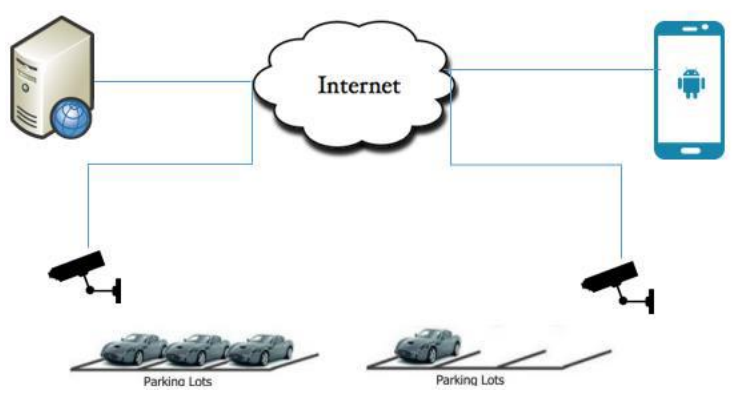

Figure 1: system architecture

In system architecture admin have a smart car. And they renting to travel. User using application they search the smart car . Using GPS and GSM module. GSM is a cellular network in that cell phones connect to it by searching for cells in the immediate vicinity. The GSM can send the data using radio wave. 


\section{International Journal of Advanced Research in Computer and Communication Engineering}

Vol. 8, Issue 1, January 2019

\section{CONCLUSION}

In this way we will implement the renting of car which is user friendly for owner and the user. who is going to rent the car via an app and is beneficial for both of them. Also tracking of car with the help of GPS and calculation of the total fare of the journey based on the status of air conditioner will be displayed to the user.

\section{REFERRENCES}

[1]. Z. Liao, "Real-time taxi dispatching using global positioning systems". Communications of the ACM, 46(5),81-83, 2003.

[2]. J. Yuan, Y. Zheng, X. Xie, and G. Sun, "T-Drive: Enhancing Driving Directions with Taxi Drivers' Intelligence," IEEE Transactions on Knowledge \& Data Engineering, vol.25, pp. 220 - 232. 2013.

[3]. M. Papageorgiou, C. Diakaki, V. Dinopoulou, A. Kotsialos, and Y. Wang, "Review of road traffic control strategies, " Proceedings of the IEEE, vol.91, pp. 2043-2067, 2003.

[4]. A. M. De Livera, R. J. Hyndman, and R. D. Snyder, "Forecasting time series with complex seasonal patterns using exponential smoothing, "Journal of the American Statistical Association, vol. 106, pp. 1513-1527,2011.

[5]. S"Robust forecasting with exponential and Holt-Winters smoothing, ". Gelper, R. Fried, and C. Croux, Journal of Forecasting, vol. 29, pp. 285$300,2010$.

[6]. V. A. Butakov and P. Ioannou, "Personalized driver assistance for signalized intersections using V2I communication," IEEE Trans. Intell.Transp. Syst., vol. 17, no. 7, pp. 1910-1919, Jul. 2016.

[7]. M. Maciejewski, "Online taxi dispatching via exact offline optimization",2014.

[8]. J. Yuan, Y. Zheng, X. Xie, and G. Sun, "T-Drive: Enhancing DrivingDirections with Taxi Drivers' Intelligence, " IEEE Transactions on Knowledge \& Data Engineering, vol 25, pp. 220 - 232. 2015.

[9]. H. Lin and D. Liu, "Study of Queue Length and Delay Calculation Based on Taxi GPS Data, " in IIth International Conference of Chinese Transportation Professionals (ICCTP), 20 I6.

[10]. C. J. Su, \& C. Y. Wu, "JADE implemented mobile multi-agent based, distributed information platform for pervasive health care monitoring". Applied Soft Computing, 11(1), pp. 315-325, 2011.

[11]. S. Gelper, R. Fried, and C. Croux, "Robust forecasting with exponential and Holt-Winters smoothing, " Journal of Forecasting, vol. 29, pp. 285$300,2010$.

[12]. C. J. Su, \& C. Y. Wu, "JADE implemented mobile multi-agent based,distributed information platform for pervasive health care monitoring". Applied Soft Computing, 11(1), pp. 315-325, 2011.

[13]. C. Wang, W. K. Ng, \& H. Chen., "From data to knowledge to action: A taxi business intelligence system". In 15 th International Conference on Information Fusion (FUSION), pp. 1623-1628, July 2012.

[14]. J. Yuan, Y. Zheng, X. Xie, and G. Sun, "T-Drive: Enhancing DrivingDirections with Taxi Drivers' Intelligence, " IEEE Transactions on Knowledge \& Data Engineering, vol 25, pp. 220 - 232. 2013.

[15]. S. Chawla, Y. Zheng, and J. Hu, "Inferring the root cause in road traffic anomalies, " in Data Mining (ICDM), 2012 IEEE 12 th International Conference on, pp. 141-150, 2012. 